\title{
Clustering and percolation in lithium borate glasses
}

\author{
Alice Vegiria) and Cristos-Platon E. Varsamis \\ Theoretical and Physical Chemistry Institute, National Hellenic Research Foundation, \\ 48 Vas. Constantinou Ave., 11635 Athens, Greece
}

(Received 30 December 2003; accepted 2 February 2004)

\begin{abstract}
Molecular dynamics simulations are carried out in $x \mathrm{Li}_{2} \mathrm{O}-(1-x) \mathrm{B}_{2} \mathrm{O}_{3}$ glasses $(x=0.2-0.6)$ at $T=1250 \mathrm{~K}$, where cluster size distributions for Li cations and nonbridging oxygen (NBO) atoms are calculated. The existence of percolating clusters above $x=0.3$ places the percolation threshold between $x=0.3$ and 0.4 for the system under investigation, which is consistent with the abrupt increase of the diffusion coefficient of $\mathrm{Li}$ cations observed at $x=0.4$. It is also shown that the clusters of Li cations consist mainly of Li atoms found in the vicinity of NBO atoms. This result explains the higher mobility exhibited by this type of cations compared to the mobility of Li cations in the vicinity of bridging oxygen atoms. (C) 2004 American Institute of Physics.
\end{abstract}

[DOI: 10.1063/1.1689644]

\section{INTRODUCTION}

Nowadays, ionic glasses constitute potential materials with considerable technological importance. The variation of room-temperature de conductivity from $10^{-15}$ up to $10^{-1} \mathrm{~S} / \mathrm{cm}$ renders them suitable for a wide range of electrical and electrochemical applications. ${ }^{1,2}$ In this context, the detailed knowledge of ionic transport properties and their dependence on glass microstructure acquires a crucial role and is, in fact, a subject of continuous scientific interest. Despite the long-standing research efforts on this topic, a general theory for ionic conductivity in a disorder medium is still lacking. ${ }^{3,4}$

Theoretical and phenomenological models proposed to explain ionic transport in glasses involve the microstructure in general and the distribution of sites hosting mobile ions. The continuous random network (CRN) model of Zachariasen ${ }^{5}$ assumes that cations are randomly, or homogeneously, distributed in voids and sites of the disordered glassy network. More recent developments put an emphasis on the intermediate-range order and give evidence of a microstructure characterized by the microsegregation of glassmodifying cations from the sublattice of the glass-forming oxide. In this modified random network (MRN) model, developed originally by Greaves for alkali silicate glasses, alkalis are proposed to form clusters, which join to create channels at high alkali concentrations. ${ }^{6}$

It is interesting to note that inhomogeneous transport dynamics involving the formation of favored pathways, or pathway clusters of cations suitable for ion migration, was also invoked in other models, like the diffusion pathway model, ${ }^{7,8}$ the cluster model, ${ }^{9-11}$ the cluster-bypass model, ${ }^{12,13}$ and the dynamic structure model. ${ }^{14,15}$ The formation of channels suitable for ion migration-i.e., percolating clusters of cations-was also visualized in previous molecular dynamics (MD) calculations of alkali silicate ${ }^{16-19}$ and borate ${ }^{20,21}$

\footnotetext{
a) Author to whom correspondence should be addressed. Electronic mail: avegiri@eie.gr
}

glasses and in reverse Monte Carlo structural models of silver ion conducting glasses and crystals. ${ }^{22,23}$ Finally, the formation of clusters, which eventually percolate above a certain concentration, was deduced from the analysis of highfrequency dielectric spectra in Li-containing borate glasses. ${ }^{24}$

Besides the spatial distribution of cations in the glassy matrix, the nature of the cation-hosting environments is an equally important aspect for the investigation of transport properties. Far-infrared and dielectric spectroscopic data revealed the existence of at least two distinct environments of cation hosting sites in ionic glasses. ${ }^{25,26}$ This "two-site" model $^{25}$ for metal ions was invoked in conductivity mechanisms for single ${ }^{27-29}$ and mixed d $^{30,31}$ alkali glasses. Furthermore, our recent molecular dynamics simulation in lithium borate glasses ${ }^{20,21,32}$ corroborated the distinction in two types of $\mathrm{Li}$ cation hosting sites: the first type of site is formed by bridging oxygen (BO) atoms of the network (b site), whereas the second one involves also the participation of nonbridging oxygen (NBO) atoms (nb site). Accordingly, a Li cation was labeled $\mathrm{Li}^{\mathrm{b}}$ or $\mathrm{Li}^{\mathrm{nb}}$ if it was found to reside predominantly in b type or nb type of sites, respectively. This distinction was clearly reflected on the different behavior exhibited by the two types of cations in their short- and long-time dynamics.

In previous work, ${ }^{20}$ we observed that in $x \mathrm{Li}_{2} \mathrm{O}-(1$ $-x) \mathrm{B}_{2} \mathrm{O}_{3}$ glasses a jump in the self-diffusion coefficient of the $\mathrm{Li}$ cations, $D_{\mathrm{Li}}$, is taking place at concentrations $x$ greater than $x=0.3$ and at $T=1250 \mathrm{~K}$. This is shown in Fig. 1(a), which is reproduced from Ref. 20. In the same figure the relative population of $\mathrm{Li}^{\mathrm{nb}}$ ions is also included. As illustrated, $D_{\mathrm{Li}}$ and the relative population of $\mathrm{Li}^{\mathrm{nb}}$ cations follow a similar dependence on the lithium oxide concentration. The variation of the dc conductivity with lithium oxide content $x$ is illustrated in Fig. 1(b) in a double-logarithmic plot. As shown, the data follow to a very good approximation a straight line, which implies a power-law dependence of conductivity on composition, of the form $\sigma_{\mathrm{dc}} \propto x^{t}$, in agreement with the dynamic structure model (DSM) (Refs. 14 and 15) and other analytical models based on a statistical distribution 

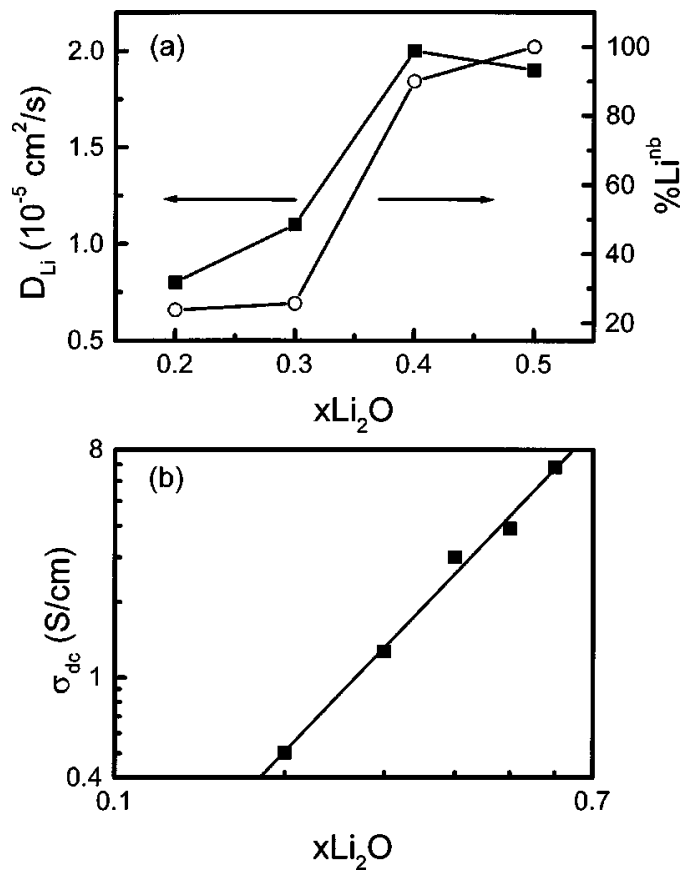

FIG. 1. (a) Diffusion coefficient of Li cations, $D_{\mathrm{Li}}$, and percentages of $\mathrm{Li}^{\text {nb }}$ ions at $1250 \mathrm{~K}$ in $x \mathrm{Li}_{2} \mathrm{O}-(1-x) \mathrm{B}_{2} \mathrm{O}_{3}$ glasses. Lines are drawn to guide the eye. (b) dc conductivity values at $T=1250 \mathrm{~K}$ in a $\log -\log$ plot. The line is a power-law least-squares fit.

of energy barrier heights ${ }^{33}$ or potential wells $\mathrm{s}^{34,35}$ over which the ions can hop.

The observed jump in $D_{\mathrm{Li}}$ was qualitatively explained through the formation of regions rich in $\mathrm{Li} / \mathrm{NBO}$ with increasing lithium oxide content. ${ }^{20}$ It was argued that these regions, rather than being isolated, as is the case at low $\mathrm{Li}_{2} \mathrm{O}$ concentrations, start to merge progressively, thereby leading to the formation of microchannels suitable for ion migration. This picture, first suggested by Greaves in the MRN model, ${ }^{6}$ was visualized in a representative snapshot of the simulated atomic structure of the $0.5 \mathrm{Li}_{2} \mathrm{O}-(1-0.5) \mathrm{B}_{2} \mathrm{O}_{3}$ glass in Refs. 20 and 21. Ionic transport along percolation pathways and the enhancement of the dc conductivity can be considered as two closely related effects. ${ }^{14,15,34}$

The purpose of the present paper is to associate the observed jump in the self-diffusion coefficient at a particular concentration with the appearance for first time of the percolation cluster. Although microchannels in the manner of Greaves have been proposed to account for the structural and transport properties in silicate glasses and have been visualized in MD simulations, ${ }^{16}$ their relationship with the predictions of a percolative type of transport has never been explicitly shown.

On these grounds, based on our previous molecular dynamics study in Li-containing borate glasses, $x \mathrm{Li}_{2} \mathrm{O}-(1$ $-x) \mathrm{B}_{2} \mathrm{O}_{3}$ (Refs. 20, 21 and 32), we proceed into a detailed numerical investigation of the properties and statistics of the clusters formed in the network either by $\mathrm{Li}$ ions or NBO atoms. In particular, we focus on the cluster distribution of either NBO atoms or $\mathrm{Li}$ cations as a function of the $\mathrm{Li}_{2} \mathrm{O}$ concentration. Moreover, we investigate the nature of the $\mathrm{Li}$ ions $\left(\mathrm{Li}^{\mathrm{b}}\right.$ or $\left.\mathrm{Li}^{\mathrm{nb}}\right)$ that constitute the resulting clusters in the network. This information will further elucidate the unique role of NBO atoms to the transport properties of this peculiar glassy system. The peculiarity stems from the fact that the charge is located either in NBO containing units or in equally charged tetrahedral units.

Finally, we should like to emphasize that it is not our intention to identify the exact position of the percolation threshold, since this would not add anything to the main purpose of this work.

\section{COMPUTATIONAL METHOD}

Molecular dynamics simulations were performed in the $x \mathrm{Li}_{2} \mathrm{O} \cdot(1-x) \mathrm{B}_{2} \mathrm{O}_{3}$ system for $x=0.2,0.3,0.4,0.5$, and 0.6 at $T=1250 \mathrm{~K}$ (Ref. 20). In all simulations, configurations consisting of ca. 256 atoms in a primitive cubic cell have been generated. The number of atoms used in each composition and the lattice constant of the unit cell are determined from experimental density data, as reported in detail in Ref. 20. Interatomic interactions were treated by means of the usual Born-Mayer-Huggins potential augmented by an additional three-body harmonic term for the $\mathrm{O}-\mathrm{B}-\mathrm{O}$ angles. The potential form and the values of the relevant parameters have been taken from Ref. 36, where a partial screening of the electrostatic charges, necessary to reproduce the experimental infrared and Raman spectra, was adopted.

Our simulations were carried out in the microcanonical ensemble, where the initial configuration was generated from a random distribution of atoms over the sites of an fcc cubic lattice. Velocities were taken from a Maxwellian distribution at $6000 \mathrm{~K}$. Periodic boundary conditions were applied and long-range Coulombic forces were handled by employing the Ewald summation method ${ }^{37}$ A fifth-order Gear predictorcorrector integrator with a time step of $0.96 \mathrm{fs}$ was used. The generated melts were quenched to the final temperature in five cooling cycles. Each cycle consisted of a fast cooling step of about 2 ps and an equilibration period of about 10 ps. The equilibration period in the final cooling cycle was $30 \mathrm{ps,}$ whereas properties were accumulated for a final period of about 180 ps.

At each time step we identified and labeled the NBO atoms in the network and the mobile $\mathrm{Li}^{\mathrm{nb}}$ and $\mathrm{Li}^{\mathrm{b}}$ cations. As in previous work, ${ }^{20}$ an oxygen atom is labeled NBO if no other boron atom is found within a sphere of ca. $2 \AA$ radius, which corresponds to the first minimum of the $\mathrm{B}-\mathrm{O}$ radial distribution function (RDF). Accordingly, a Li cation is labeled $\mathrm{Li}^{\mathrm{nb}}$ if it is found close to an NBO atom and $\mathrm{Li}^{\mathrm{b}}$ if it is close to a bridging oxygen atom of a tetrahedral borate unit, with the $\mathrm{Li}-\mathrm{O}$ distance being less than or equal to the first maximum of the $\mathrm{Li}-\mathrm{O}$ RDF.

We used this information in order to calculate at each time step the number of isolated clusters in the glass consisting of either NBO atoms or of all Li cations. NBO atoms are taken into account due to their important role in the diffusion of $\mathrm{Li}$ cations. ${ }^{20,24,32,38}$ Specifically, we have shown that $\mathrm{Li}$ cations that are embedded in nb type of sites exhibit enhanced mobilities compared to those found in b type of sites.

In order to evaluate the number of isolated clusters, we created for each particle (NBO or $\mathrm{Li}$ ) a list that contained all of its first neighbors by applying a distance criterion accord- 
ing to the corresponding $\mathrm{NBO}-\mathrm{NBO}$ and $\mathrm{Li}-\mathrm{Li}$ RDFs. Thus particles that were found in a distance less than or equal to the first maximum of the corresponding RDF were included in the neighbors list of each $\mathrm{NBO}$ or $\mathrm{Li}$ particle. In the case of NBO lists, this criterion was applied by taking into account the second sharp peak of the corresponding NBONBO RDF as illustrated in Fig. 4 of Ref. 20, since the first peak corresponds to the correlation of the NBO atoms found in the same triangular borate unit. The algorithm for the generation of final lists consisting of isolated clusters of any size $(s)$ was based on the successive merging of pairs of neighbors' lists, which contained at least one common element. The new created lists were compared again in pairs in an iterative cycle, until no common elements between two resultant lists were found. Thus for each concentration the number of clusters of $\mathrm{NBO}$ or $\mathrm{Li}$ particles $\left[N_{s}^{\mathrm{NBO}}(s)\right.$ or $\left.N_{s}^{\mathrm{Li}}(s)\right]$ of size $s$ as a function of $s$ were generated.

Similarly, we calculated the probability that a cluster of size $(s)$ is a percolating cluster for either NBOs or Li particles. In doing so, at each time step, a cluster of size $s$ is denoted as the percolation cluster if at least two atoms in the cluster are found at two opposite faces of the primitive cell, within a certain distance from the boundaries. This distance was taken as the half of the first maximum of the corresponding NBO-NBO or Li-Li RDFs.

\section{RESULTS AND DISCUSSION}

The calculated time-averaged distributions of the number of clusters of NBO atoms, $\left\langle N_{s}^{\mathrm{NBO}}(s)\right\rangle$, as a function of cluster size $(s)$ for all concentrations are displayed in Figs. 2(a) and 2(b). Note that the cluster size distribution curves in Fig. 2(a) are for concentrations $x=0.2$ and 0.3 in a semilogarithmic scale, whereas in Fig. 2(b) are for $x=0.4,0.5$, and 0.6 in a double-logarithmic scale. We verified that the total number of NBO atoms, calculated for each composition by integrating the corresponding distribution curves, coincides perfectly with the one obtained by simply counting NBO atoms in the simulation box.

If we take into account the theoretical predictions of standard percolation theory, ${ }^{39}$ the cluster size distribution will have to decay exponentially below a critical concentration, which can be associated with the percolation threshold $p_{c}$ according to the following expression:

$$
\ln N_{s} \propto-s \text { for } p<p_{c} \text {. }
$$

At this point, we shall have to clarify that while the standard meaning of $p$ is the occupation probability of a lattice site, in the present context it can be associated with the number concentration of NBO or Li particles.

On the other hand, at $p \geqslant p_{c}$, the cluster size distribution curves should obey the equation

$$
N_{s}(p) \propto s^{-\tau} f(z),
$$

where $z \equiv\left(p-p_{c}\right) s^{\sigma}$.

At the percolation threshold, $p=p_{c}$. Here $f(z)=1$ and consequently the distribution curves follow a simple power law. The function $f(z)$ is known to be analytic and to possess a single maximum.
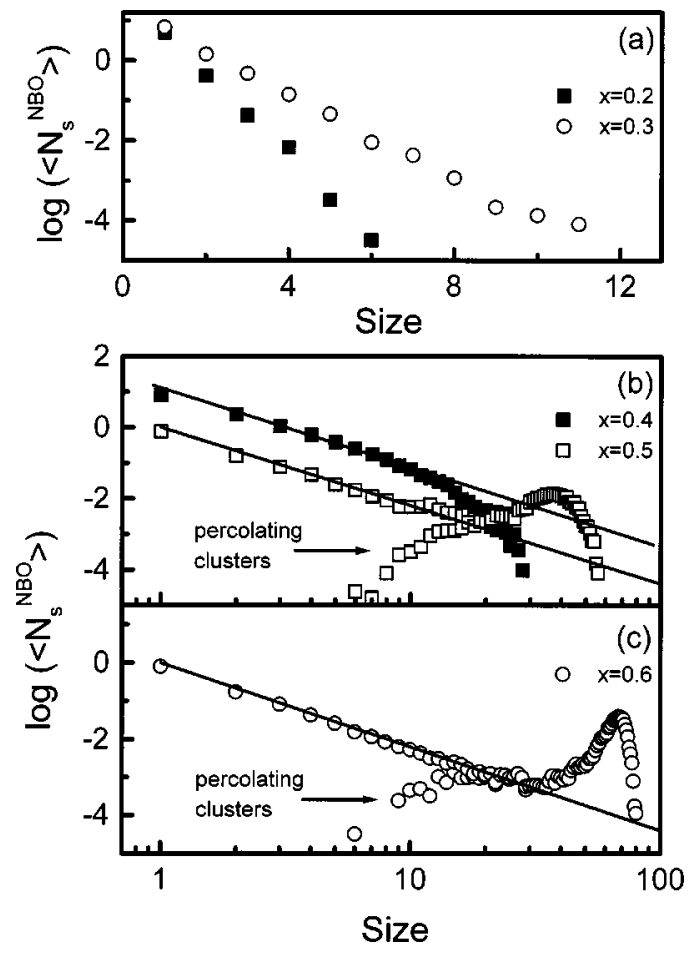

FIG. 2. (a) Time average distributions of the number of clusters of NBO atoms with size $s,\left\langle N_{s}^{\mathrm{NBO}}(s)\right\rangle$, as a function of cluster size $(s)$ for (a) $x$ $=0.2$ and 0.3 in a semilogarithmic scale, (b) $x=0.4$ and 0.5 in a log-log plot, and (c) $x=0.6$ in a log-log plot. The corresponding distribution curves for the percolating clusters only are also included. The straight lines drawn in (b) and (c) have a slope equal to 2.2.

The results reported in Fig. 2 show that the cluster size distributions are to a very good approximation exponential (straight lines) for $x=0.2$ and 0.3 , suggesting that for these compositions the system is found below the percolation threshold. On the other hand, for $x>0.3$ the cluster size distribution curves are characterized by a power law with an exponent ca. 2.2 [corresponding to the straight line in Figs. 2(b) and 2(c)] up to a cutoff size depending on concentration. In addition for $x=0.5$ and 0.6 the curves exhibit a very pronounced peak at larger cluster sizes. The particular value of the power-law exponent is in agreement with the predictions of random percolation theory, ${ }^{40,41}$ whereas the pronounced peak at the large-size region is attributed to the existence of percolating clusters. ${ }^{42}$ This is also inferred from the distributions of the percolating NBO clusters, which have been calculated independently and are superimposed in the same figure for $x=0.5$ and 0.6. It is clear that there is a perfect coincidence between the corresponding peaks in the NBO cluster size distribution and the percolating NBO cluster size distribution. Therefore, it is safe to assume that concentrations above $x=0.3$ should lie above the percolation threshold.

In a similar way, the time-averaged distributions of the number of clusters of Li cations, $\left\langle N_{s}^{\mathrm{Li}}(s)\right\rangle$, as a function of cluster size $(s)$ for all concentrations is illustrated in Figs. 3(a)-3(c). The axes scales are the same as in Fig. 2. The time-averaged distributions are again exponential for low concentrations $(x=0.2$ and 0.3$)$ and power law for higher concentrations up to a certain cutoff size. Compared to the 

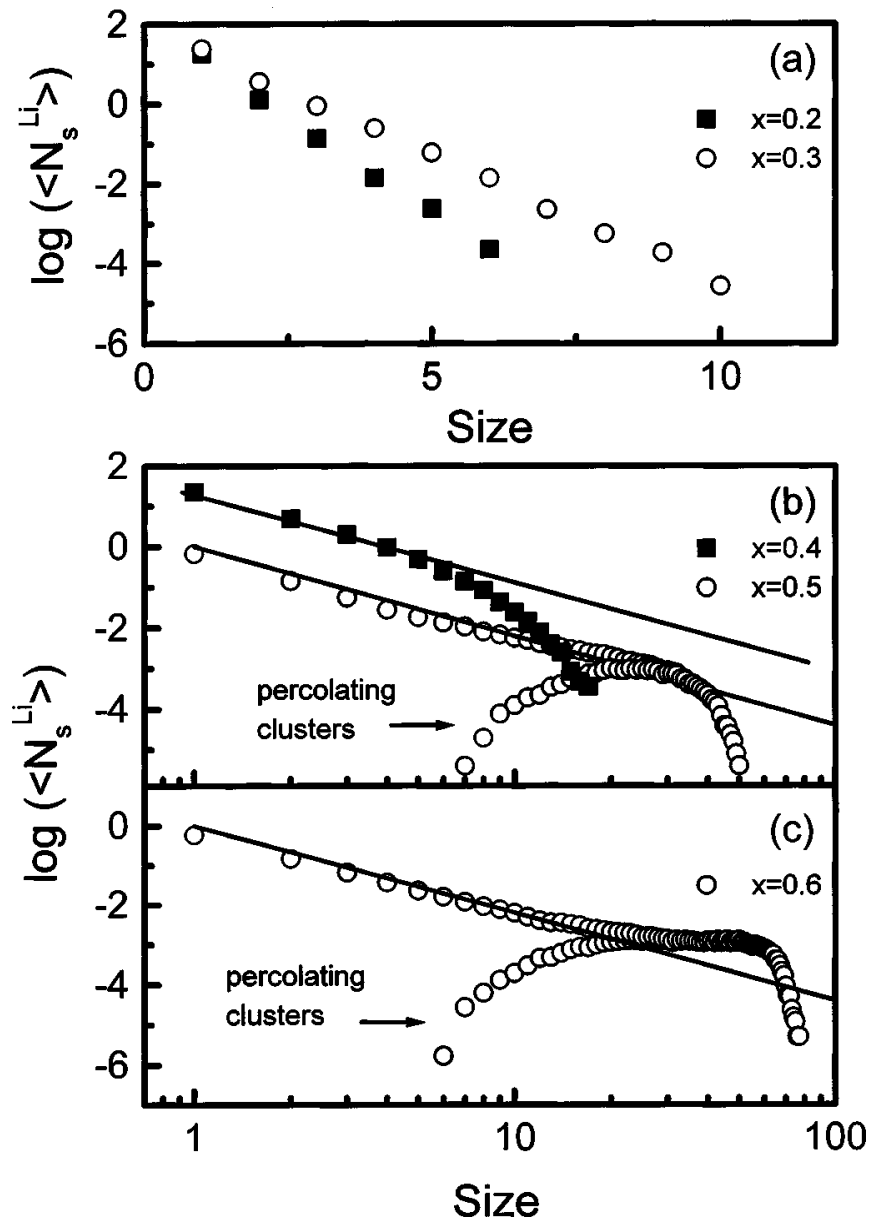

FIG. 3. As in Fig. 2, but for Li cations.

distributions of NBO-containing clusters displayed in Figs. 2(b) and 2(c) at $x=0.5$ and 0.6 , the distributions of the $\mathrm{Li}$ clusters show a rather broad shoulder than a sharp peak. This can be attributed to the different, by an order of magnitude, mobility of the NBO and Li particles, as found previously. ${ }^{20}$ Thus the time-averaged NBO cluster size distributions are less smeared out than the distributions of the clusters of the more mobile Li cations, which suffer a drastic averaging. Again, as in Figs. 2(b) and 2(c), the time-averaged size distributions of the percolating $\mathrm{Li}$ clusters coincide perfectly well with the peaks at high $s$ values of the distributions of $\mathrm{Li}$ clusters.

The aforementioned results clearly show that the observed jump in the self-diffusion coefficient at $x=0.4$ can be associated with the formation of percolating clusters of NBO or Li atoms in the glassy network. This is further evidenced by calculating the probability that a cluster of size $s$ is a percolating one. The calculated probabilities are depicted in Figs. 4(a) and 4(b) for clusters of NBO and Li atoms, respectively. It is clear that in both cases for concentrations $x$ $=0.2$ and 0.3 , the probability to find a percolating cluster is practically negligible. On the contrary, for concentrations greater than $x=0.3$ this probability approaches 0.9 for cluster sizes in the range between 20 and 30 depending on concentration, placing the percolation threshold somewhere between $x=0.3$ and 0.4 .
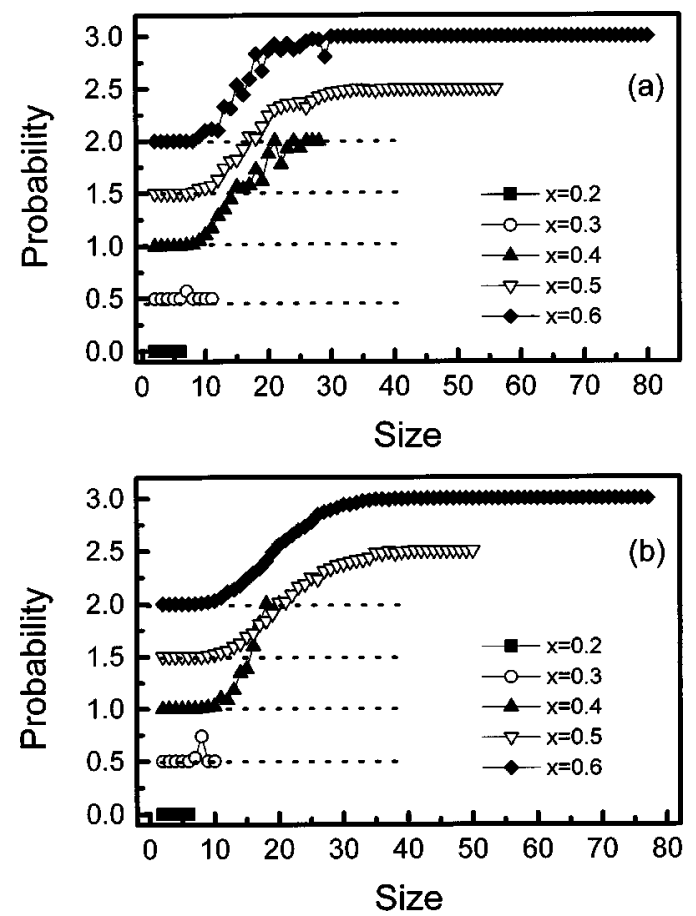

FIG. 4. Probability that a cluster of size $s$ is a percolating cluster for all compositions (a) for $\mathrm{NBO}$ atoms and (b) for $\mathrm{Li}$ cations. Notice that the curves are vertically offset by 0.5 for clarity. Dotted lines indicate the zero for the corresponding distribution curve.

The present estimation of the percolation threshold and the formation of clusters are in accordance with the interpretation of the experimental results of time domain reflectometry in the same family of glasses. ${ }^{24}$ The authors suggested that infinite percolating clusters arise at $x=0.4$ and that the percolation threshold can be most probably traced at $x$ $=0.42$. Experimental evidence for the existence of percolation pathways for diffusion has been provided by NMR spin-lattice relaxation studies which estimated the percolation threshold between 15 and $22 \mathrm{~mol} \% \mathrm{Na}_{2} \mathrm{O}$ in $x \mathrm{Na}_{2} \mathrm{O}-(1-x) \mathrm{B}_{2} \mathrm{O}_{3}$ glasses. ${ }^{43}$

An estimation of the spatial extent of a cluster of size $s$ can be obtained from the calculation of its spanning length $\ell$, defined as the maximum separation of two sites in the cluster, according to the relation

$$
\ell=\max \left|\vec{r}_{i}-\vec{r}_{j}\right| \text {. }
$$

The calculated curves of the spanning length versus cluster size for NBO clusters are shown in Fig. 5 in doublelogarithmic scale. The relationship between the cluster size $s$ and the spanning length $\ell$ is described by a power law $\ell \propto s^{1 / d}$, where $d$ denotes the dimensionality of the cluster. It is observed that for low alkali concentrations below the estimated percolation threshold these curves are straight lines with a slope very close to $1(d \sim 1.2)$, which is a reasonable result for such small clusters consisting of a few particles only. Inspection of the magnitude of their spanning length shows that the particles in these clusters do not form linear chains, as would be expected in a meta-borate crystalline configuration, where the single NBO atoms of borate triangles are arranged into chains. ${ }^{44}$ The spanning length for low concentrations is systematically much less than the value 


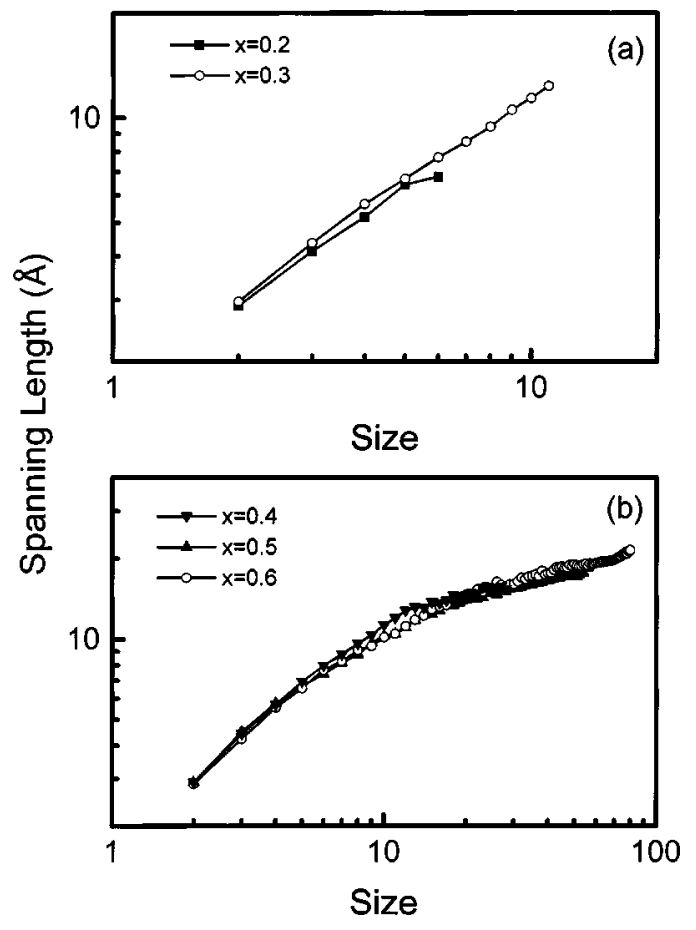

FIG. 5. Spanning length of NBO clusters as a function of their size $s$ in a $\log -\log$ plot (a) for $x=0.2$ and 0.3 and (b) for $x=0.4,0.5$, and 0.6 .

it would have in a linear-chain-type arrangement. It is also observed that the spanning length never exceeds the linear dimension of our simulation box at ca. $14 \AA$, which is consistent with our estimation that the percolation threshold should be above $x=0.3$.

Regarding the spanning length variation of the clusters for concentrations larger than $x=0.3$, a curved rather than a straight line is observed, due to the finite size of the box. Therefore, the dimensionality of the cluster cannot be calculated in this way. The spanning length for these concentrations exceeds the linear dimension of the box for a cluster size of about 25 . This is consistent with our previous observation reported in Fig. 4(a) - that a cluster of size above 25 is a percolating cluster with a probability approaching 1 .

Our following step is to identify the particular type of cations, which constitute a Li cluster of size $s$. At every time step, we distinguish whether a Li cation is of nb or b type, and it is examined whether it belongs to a particular isolated cluster of size $s$. In this way, we were able to calculate their relative population in the clusters. The probability that a $\mathrm{Li}^{\mathrm{nb}}$ cation is a member of a cluster of size $s$ is displayed in Fig. 6 , for all concentrations. For small concentrations this probability amounts to about 0.8 or 0.9 . Only the isolated cations can be with an almost equal probability either of nb or $b$ type. On the other hand, the probability that a cluster consists of $\mathrm{Li}^{\mathrm{b}}$ cations is negligibly small. Put in other words, $\mathrm{Li}^{\mathrm{b}}$ cations are somehow isolated, whereas $\mathrm{Li}^{\mathrm{nb}}$ cations tend to agglomerate. For larger concentrations, the majority of $\mathrm{Li}$ ions are of nb type [see Fig. 1(a)]. Therefore this probability is very close to one. Since by definition a $\mathrm{Li}^{\mathrm{nb}}$ cation can be found close to an NBO atom only, the glassy network consists of regions, which are rich in $\mathrm{NBO}$ and $\mathrm{Li}^{\mathrm{nb}}$ particles, even at concentrations below the percolation threshold. This

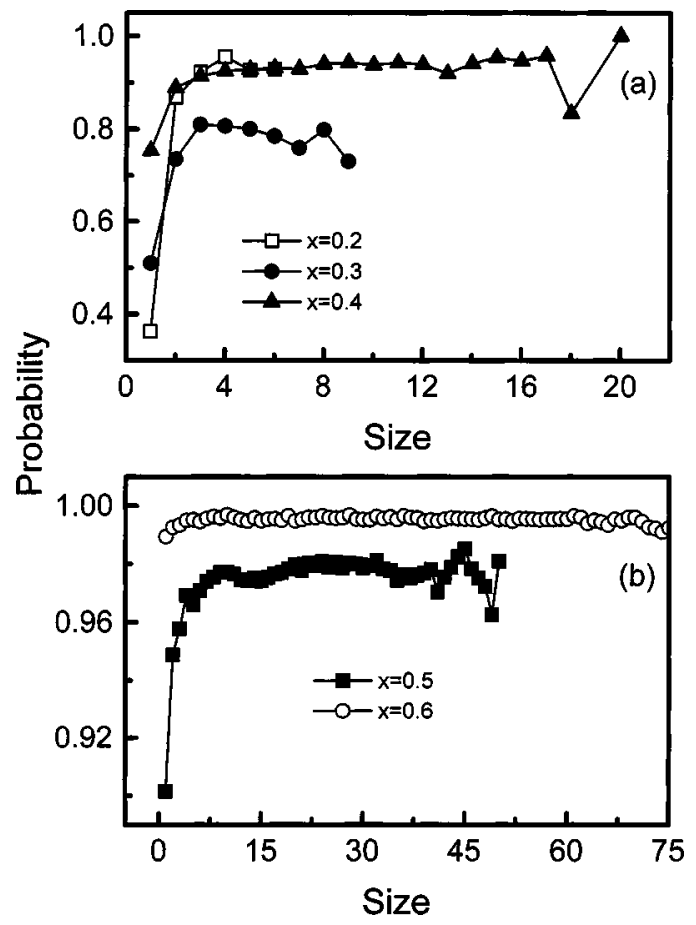

FIG. 6. Probability that a $\mathrm{Li}^{\mathrm{nb}}$ cation is a part of a cluster of size $s$ (a) for $x=0.2,0.3$, and 0.4 and (b) for $x=0.5$ and 0.6 .

$\mathrm{Li} / \mathrm{NBO}$ aggregation provides a plausible explanation for the larger diffusion coefficient of the $\mathrm{Li}^{\mathrm{nb}}$ cations observed in Ref. 20.

In order to further investigate the spatial distribution of both $\mathrm{NBO}$ and $\mathrm{Li}$ atoms in the network we compare their first neighbors' distances derived from the first peak of the corresponding pair radial distribution functions $R_{\mathrm{RDF}}$, with the distances expected from a three-dimensional homogeneous distribution $R_{\mathrm{HOM}}$ of these particles in the simulation box. The distance values are reported in Table I. It is observed that for all concentrations $R_{\mathrm{RDF}}$ is systematically smaller than $R_{\text {HOM }}$, implying an inhomogeneous distribution of $\mathrm{Li}$ and NBO atoms in the borate network. This structural picture is consistent with the modified random network model. ${ }^{6}$

It is worth noting that the $R_{\mathrm{RDF}}$ distance for $\mathrm{NBO}$ atoms remains constant, independent of composition. This means that the NBO atoms are created in neighboring sites even at low $\mathrm{Li}_{2} \mathrm{O}$ concentrations where the NBO population is very small. In this way, we can visualize the way the percolating channels are formed-namely, from the progressive evolution of small isolated droplets of NBO atoms to larger ones,

TABLE I. First neighbors' distances in $\AA$ for $\mathrm{Li}^{+}$and NBO atoms, derived from the RDF functions $\left(R_{\mathrm{RDF}}\right)$ and by assuming homogeneous distribution of these atoms in the box $\left(R_{\mathrm{HOM}}\right)$.

\begin{tabular}{cccccc}
\hline \hline & \multicolumn{3}{c}{$\mathrm{Li}$} & & \multicolumn{2}{c}{$\mathrm{NBO}$} \\
\cline { 2 - 3 } \cline { 5 - 6 }$x$ & $R_{\mathrm{HOM}}(\AA)$ & $R_{\text {RDF }}(\AA)$ & & $R_{\text {HOM }}(\AA)$ & $R_{\text {RDF }}(\AA)$ \\
\hline 0.3 & 4.8 & 3.3 & & 6.9 & 3.2 \\
0.4 & 4.4 & 3.1 & & 5.4 & 3.2 \\
0.5 & 4.0 & 3.0 & & 4.5 & 3.2 \\
\hline \hline
\end{tabular}




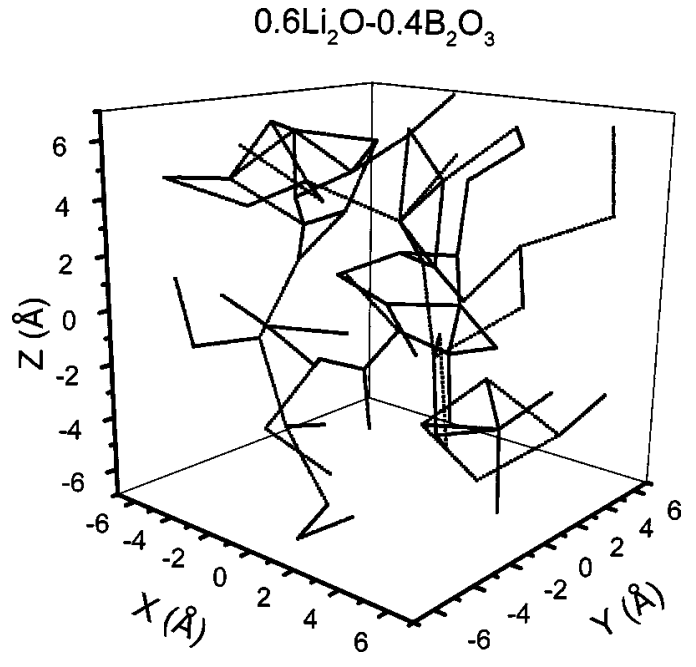

FIG. 7. Snapshot of a percolating cluster consisting of NBO atoms of size $s=70$ for the $0.6 \mathrm{Li}_{2} \mathrm{O}-0.4 \mathrm{~B}_{2} \mathrm{O}_{3}$ composition. Straight lines connect NBO atoms situated at the intersection of two or more such lines.

up to a critical concentration, where these droplets merge to the percolating cluster. Typical snapshots for the $s=70$ and 30 NBO-percolating clusters are shown in Figs. 7 and 8, respectively, for the $0.6 \mathrm{Li}_{2} \mathrm{O}-0.4 \mathrm{~B}_{2} \mathrm{O}_{3}$ composition. A similar picture is obtained for the Li-percolating clusters as well. As shown, the NBO particles are not arranged in a compact form, but they ramify through the simulation box. The lines in Figs. 7 and 8 connect particles that are found within the first neighbors' shell and serve as a guide for the eye. The particles are situated on the intersection of these lines.

\section{CONCLUSIONS}

The spatial distributions of the $\mathrm{Li}$ cations and nonbridging oxygen atoms in $x \mathrm{Li}_{2} \mathrm{O}-(1-x) \mathrm{B}_{2} \mathrm{O}_{3}$ glasses for $x$ $=0.2,0.3,0.4,0.5$, and 0.6 are investigated at $T=1250 \mathrm{~K}$ by means of molecular dynamics simulations. The calculated time-averaged cluster size distribution curves imply a clustered structure of $\mathrm{Li}$ and $\mathrm{NBO}$ particles in the glassy net-

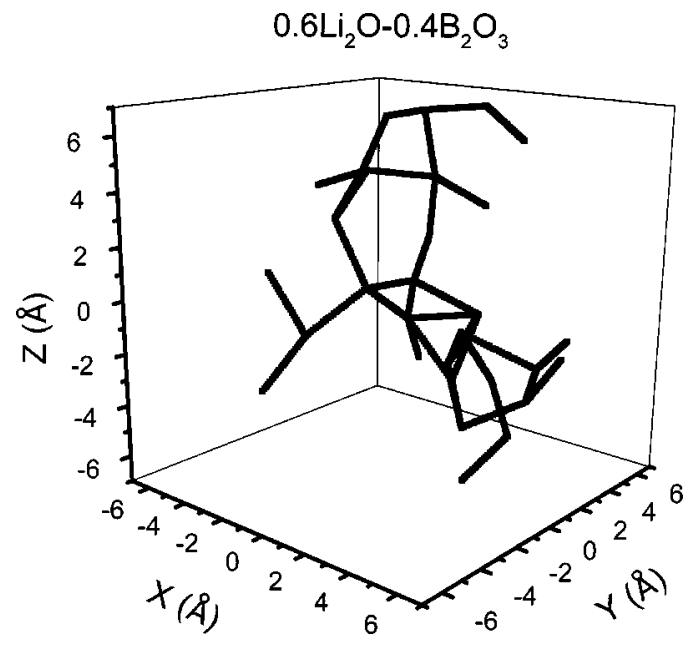

FIG. 8. Snapshot of a percolating cluster consisting of NBO atoms of size $s=30$ for the $0.6 \mathrm{Li}_{2} \mathrm{O}-0.4 \mathrm{~B}_{2} \mathrm{O}_{3}$ composition. Straight lines connect NBO atoms situated at the intersection of two or more such lines. work. The largest cluster size increases with $\mathrm{Li}_{2} \mathrm{O}$ content. The appearance of percolating clusters at $x=0.4$ places the percolation threshold for this family of glasses somewhere between $x=0.3$ and 0.4 , in close agreement with previous experimental results. ${ }^{24}$ Moreover, it explains the sharp increase of the diffusion coefficients of Li cations observed in our previous work for $x>0.3$ (Ref. 20). In addition, the predominant constitution of the Li clusters by cations of the $\mathrm{nb}$ type gives an explanation for their higher diffusivity.

It is further shown that the $\mathrm{Li}$ and $\mathrm{NBO}$ atoms follow an inhomogeneous spatial distribution in the glassy network, which consists of regions enriched with $\mathrm{NBO}$ and $\mathrm{Li}^{\mathrm{nb}}$ particles, even at concentrations below the percolation threshold. This spatial inhomogeneity is very well reflected on the dynamic heterogeneity of $\mathrm{Li}$ cations as evidenced by the higher diffusion coefficient of the $\mathrm{Li}^{\mathrm{nb}}$ cations observed in Ref. 20.

\section{ACKNOWLEDGMENT}

We thank Dr. E. I. Kamitsos for helpful discussions.

${ }^{1}$ T. Minami, J. Non-Cryst. Solids 95\&96, 107 (1987).

${ }^{2}$ M. D. Ingram, Curr. Opin. Solid State Mater. Sci. 2, 399 (1997).

${ }^{3}$ A. Hunt, J. Non-Cryst. Solids 220, 1 (1997).

${ }^{4}$ C. A. Angell, Chem. Rev. 90, 523 (1990).

${ }^{5}$ W. H. Zachariasen, J. Am. Chem. Soc. 54, 3841 (1932).

${ }^{6}$ G. N. Greaves, J. Non-Cryst. Solids 71, 203 (1985).

${ }^{7}$ G. Carini, Phys. Rev. B 29, 3567 (1984).

${ }^{8}$ T. Minami, J. Non-Cryst. Solids 73, 273 (1985).

${ }^{9}$ M. Tachez, A. J. Dianoux, J. P. Malugani, and R. Mercier, Solid State Ionics 18\&19, 372 (1986)

${ }^{10}$ A. Fontana, F. Roca, and M. P. Fontana, Phys. Rev. Lett. 58, 503 (1987).

${ }^{11}$ C. Rousselot, J. P. Malugani, R. Mercier, M. Tachez, P. Chieux, A. J. Pappin, and M. D. Ingram, Solid State Ionics 78, 211 (1995).

${ }^{12}$ M. D. Ingram, M. A. Mackenzie, W. Muller, and M. Torge, Solid State Ionics 28-30, 677 (1988)

${ }^{13}$ M. D. Ingram, Philos. Mag. B 60, 729 (1989).

${ }^{14}$ A. Bunde, M. D. Ingram, and P. Maass, J. Non-Cryst. Solids 172-174, 1222 (1994)

${ }^{15}$ P. Maass, A. Bunde, and M. D. Ingram, Phys. Rev. Lett. 68, 3064 (1992).

${ }^{16}$ C. Huang and A. N. Cormack, J. Chem. Phys. 93, 8180 (1990).

${ }^{17}$ H. Melman and S. Garofalini, J. Non-Cryst. Solids 134, 107 (1991).

${ }^{18}$ B. Vessal, G. N. Greaves, P. T. Marten, A. V. Chadwick, R. Mole, and S. Houde-Walter, Nature (London) 356, 504 (1992).

${ }^{19}$ J. Oviedo and J. F. Sanz, Phys. Rev. B 58, 9047 (1998).

${ }^{20}$ C. P. E. Varsamis, A. Vegiri, and E. I. Kamitsos, Phys. Rev. B 65, 104203 (2002).

${ }^{21}$ C. P. E. Varsamis, A. Vegiri, and E. I. Kamitsos, Condens. Matter Phys. 4, 119 (2001).

${ }^{22}$ St. Adams and J. Swenson, Phys. Rev. Lett. 84, 4144 (2000).

${ }^{23}$ S. Adams and J. Swenson, Phys. Rev. B 63, 054201 (2001).

${ }^{24}$ A. H. Verhoef and H. W. den Hartog, Solid State Ionics 68, 305 (1994).

${ }^{25}$ E. I. Kamitsos and G. D. Chryssikos, Solid State Ionics 105, 75 (1998) and references therein.

${ }^{26}$ S. Devautour, C. P. E. Varsamis, F. Henn, E. I. Kamitsos, J. C. Giuntini, J. V. Zanchetta, and J. Vanderschueren, J. Phys. Chem. B 105, 5657 (2001).

${ }^{27}$ R. J. Elliott, L. Perondi, and R. A. Barrio, J. Non-Cryst. Solids 168, 167 (1994).

${ }^{28}$ M. D. Ingram, J. Non-Cryst. Solids 222, 42 (1997).

${ }^{29}$ R. A. Montani, Solid State Ionics 89, 287 (1996).

${ }^{30}$ R. A. Montani, J. Non-Cryst. Solids 215, 307 (1997).

${ }^{31}$ B. H. Schulz, M. Dubiel, and M. Schulz, J. Non-Cryst. Solids 241, 149 (1998).

${ }^{32}$ C. P. E. Varsamis, A. Vegiri, and E. I. Kamitsos, J. Non-Cryst. Solids 307-310, 956 (2002).

${ }^{33}$ S. D. Baranovskii and H. Cordes, J. Chem. Phys. 111, 7546 (1999).

${ }^{34}$ A. Hunt, J. Non-Cryst. Solids 175, 59 (1994). 
${ }^{35}$ S. Sen, A. M. George, and J. F. Stebbins, J. Non-Cryst. Solids 197, 53 (1996).

${ }^{36}$ A. H. Verhoef and H. W. den Hartog, J. Non-Cryst. Solids 182, 235 (1995).

${ }^{37}$ M. P. Allen and D. J. Tildesley, Computer Simulations of Liquids (Clarendon, Oxford, 1986), p. 156

${ }^{38}$ A. Karthikeyan, P. Vinatier, A. Levasseur, and K. J. Rao, J. Phys. Chem. B 103, 6185 (1999).
${ }^{39}$ D. Stauffer, Phys. Rep. 54, 1 (1979).

${ }^{40}$ N. Jan, Physica A 266, 72 (1999).

${ }^{41}$ A. Oleinikova, I. Brovchenko, A. Geiger, and B. Guillot, J. Chem. Phys. 117, 3296 (2002)

${ }^{42}$ J. Rudnick, P. Nakmahachalasint, and G. Gaspari, Phys. Rev. E 58, 5596 (1998).

${ }^{43}$ S. Sen and J. F. Stebbins, Phys. Rev. B 55, 3512 (1997).

${ }^{44}$ W. H. Zachariasen, Acta Crystallogr. 17, 749 (1964). 